\title{
Characterization and Calibration of Large Area Resistive Strip Micromegas Detectors
}

Philipp Lösel, on behalf of the ATLAS Muon Collaboration

LMU Munich, Germany

\begin{abstract}
Resistive strip Micromegas detectors have been tested extensively as small detectors of about $10 \times 10 \mathrm{~cm}^{2}$ in size and they work reliably at high rates of $100 \mathrm{kHz} / \mathrm{cm}^{2}$ and above. Tracking resolution well below $100 \mu \mathrm{m}$ has been observed for $100 \mathrm{GeV}$ muons and pions. Micromegas detectors are meanwhile proposed as large area muon precision trackers of $2-3 \mathrm{~m}^{2}$ in size. To investigate possible differences between small and large detectors, a $1 \mathrm{~m}^{2}$ detector with 2048 resistive strips at a pitch of $450 \mu \mathrm{m}$ was studied in the LMU Cosmic Ray Measurement Facility (CRMF) using two $4 \times 2.2 \mathrm{~m}^{2}$ large Monitored Drift Tube (MDT) chambers for cosmic muon reference tracking. A segmentation of the resistive strip anode plane in $57.6 \mathrm{~mm}$ x $93 \mathrm{~mm}$ large areas has been realized by the readout of 128 strips with one APV 25 chip each and by eleven $93 \mathrm{~mm}$ broad trigger scintillators placed along the readout strips. This allows for mapping of homogeneity in pulse height and efficiency, determination of signal propagation along the $1 \mathrm{~m}$ long anode strips and calibration of the position of the anode strips.
\end{abstract}

Keywords: ATLAS, Micromegas, Muon Spectrometer Upgrade, resistive strips, Cosmic Ray Measurement Facility

\section{Introduction}

The high luminosity upgrade of LHC to $5-7 \times 10^{-34} \mathrm{~s}^{-1} \mathrm{~cm}^{-2}$ will increase the background hit rate to $15 \mathrm{kHz} \mathrm{cm}^{-2}$ in both inner end-caps of the ATLAS [1] muon spectrometer, the socalled Small Wheels. Currently, the Monitored Drift Tube chambers (MDT), precision muon trackers, will no longer be able to handle these background rates. Additional motivation for the New Small Wheels (NSW) is a trigger bandwidth limitation, as the endcap region is currently dominated by fake triggers, which will saturate the $\mathrm{L} 1$ trigger with moderate $p_{\mathrm{T}}$ events, requiring additional directional trigger capability. Both drawbacks will be eliminated in the NSW by the use of 16 active detection planes consisting of two quadruplets of Micromegas (MICRO MEsh GAseous Structure) as main tracking system and two quadruplets of sTGCs (small-strip Thin Gap Chambers) as main triggering system, both systems capable of precision tracking and directional triggering. [2]

Micromegas (MM) consist of three planar elements: cathode, micro mesh and anode strip readout plane [3]. Four German institutes are building the $2 \mathrm{~m}^{2} \mathrm{MMs}$, for the outer module of the small sectors (SM2) of the NSW.

\section{Cosmic Ray Measurement Facility}

The first step of the validation for ATLAS of a SM2 quadruplet will be performed in the Munich Cosmic Ray Measurement Facility (CRMF) which is equipped with two $4 \times 2.2 \mathrm{~m}^{2}$ ATLAS MDT BOS chambers for precision muon reference tracking. Two scintillator hodoscopes trigger the readout electronics

Email address: philipp. loesel@physik. uni-muenchen.de (Philipp Lösel, on behalf of the ATLAS Muon Collaboration) with a time resolution of better than $1 \mathrm{~ns}$. The goal is to demonstrate proper operation by efficiency and pulse-height maps and to recheck the mechanical precision of the quadruplets with cosmic muons.

The readout consists of three data streams, a VME based stream for the trigger, the standard ATLAS MDT GOLA-Filar data stream and the MM stream based on the RD51 Scalable Readout System (SRS) [5]. All 12288 electronic channels of an SM2 detector will be connected via 96 APV25 [4] front-end boards to the SRS. After merging the data streams, the track reconstructed by the MDTs is used as reference for the MM. Due to the angular acceptance of $-30^{\circ}$ to $+30^{\circ}$ the tracks give not only information on strip positions within the readout plane but also information on the perpendicular dimension, the vertical position of the active planes.

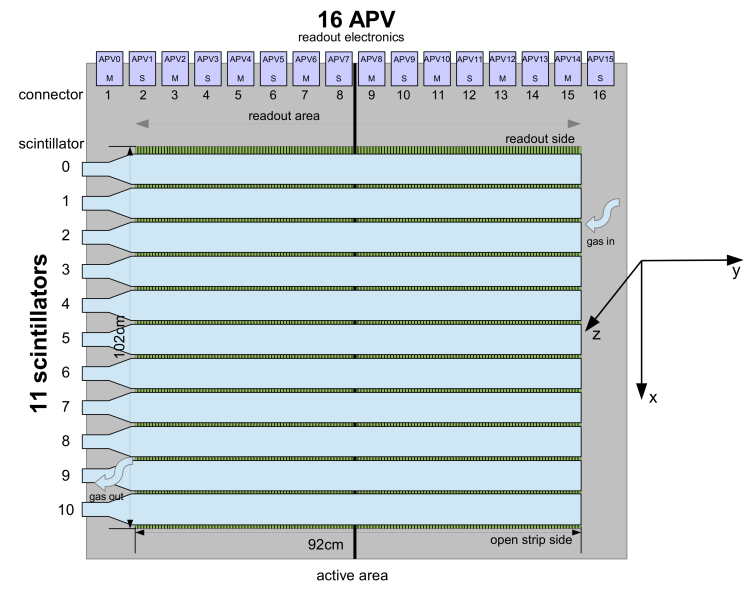

Figure 1: $92 \times 102 \mathrm{~cm}^{2}$ Mircomegas chamber divided into 176 parts (16 APV25 front-end boards $\times 11$ scintillators) 
To prepare these measurements a $102 \times 92 \mathrm{~cm}^{2} \mathrm{MM}$ with 2048 electronic channels divided into 16 APV25 front-end boards has been under investigation in the CRMF. Along the strips of this chamber eleven additional scintillators were placed to trigger only on muons traversing the MM (see figure 1). This leads to a segmentation into $176(16 \times 11)$ identical parts of about $57.6 \times 93 \mathrm{~mm}^{2}$. All following results refer to this $1 \mathrm{~m}^{2}$ chamber.

\section{Mechanical Deformations}

Using inclined tracks, the difference between predicted and reconstructed hit position in the MM, the so-called residual, allows determination of the deviation in $\mathrm{z}$ of the detector plane (see figure 2). Due to the overpressure of about 10 mbar (only 12 mbar is foreseen for ATLAS) the deviation in the middle plane of the chamber is about $0.8 \mathrm{~mm}$, the total bulging is then about $1.6 \mathrm{~mm}$, in agreement with ANSYS [6] finite element simulations. At the SM2 quadruplets six interconnections in the active area will fix the surfaces of the quadruplet at six well defined points and will thus limit bulging.

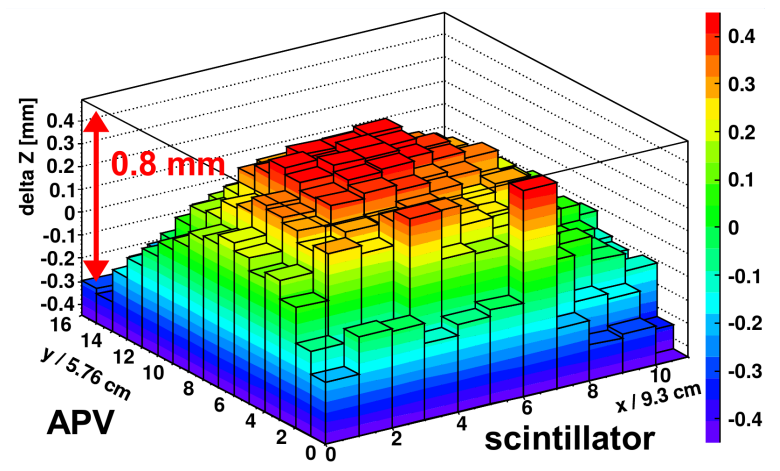

Figure 2: Deformation in $\mathrm{z}$ direction in the middle plane of the chamber of about $0.8 \mathrm{~mm}$ due to $10 \mathrm{mbar}$ overpressure

Figure 3 shows the variation of the mean value of the residual distribution. The detector is about $4 \mathrm{~mm}$ tilted in y direction and shows a step between its two readout PCB sheets due to misalignment during the gluing process. The difference $\delta y$ starts at $0.1 \mathrm{~mm}$ and goes up to $0.5 \mathrm{~mm}$ and is explained in figure 4 .

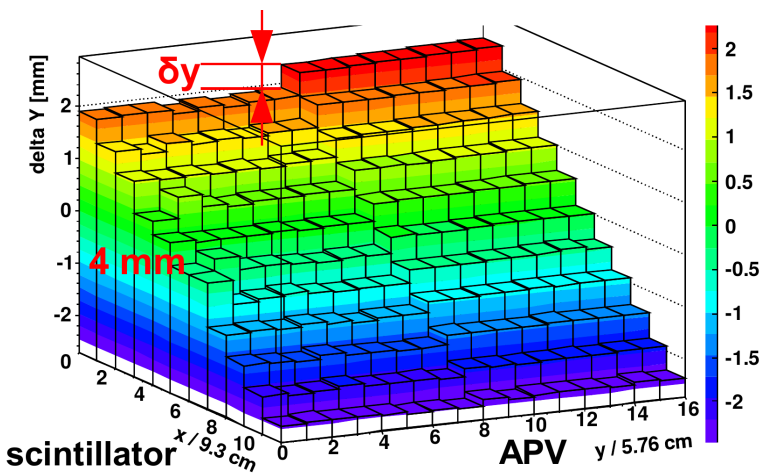

Figure 3: Non-homogeneous shift $\delta \mathrm{y}$ between the two readout boards due to non-monitored gluing process

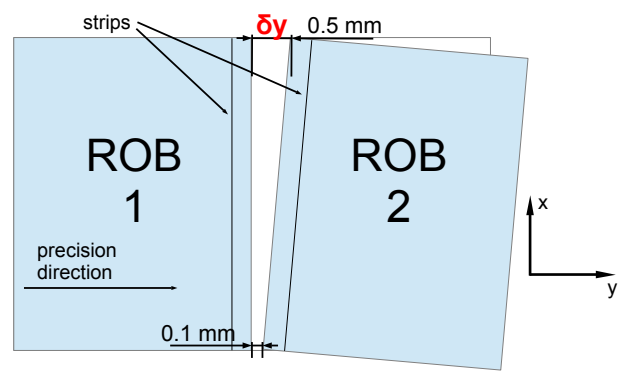

Figure 4: Sketch of the displacement $\delta y$ between the two readout boards

\section{Results of Studies Using a $1 \mathrm{~m}^{2}$ Micromegas Prototype}

Additionally, for all segments of the $1 \mathrm{~m}^{2} \mathrm{MM}$ the efficiency and pulse height distribution was determined. Both show a rather homogeneous behavior over the whole active area with an efficiency of $(94.9 \pm 0.9) \%$ and a pulse height distribution varying by about $10 \%$ RMS only.

Measurements with cosmic muons in the CRMF reach spatial resolutions of about $300 \mu \mathrm{m}$. This is dominated by the multiple scattering of the low-energy cosmic muons and corresponds to a spatial resolution of $83 \mu \mathrm{m}$ for the same detector using high energy $120 \mathrm{GeV}$ pion beams at H6 (SPS/CERN).

Using the electron drift information as a function of the responding readout-strip allows for a TPC-like analysis method. Here, the track angle of the traversing muons have been reconstructed with an angular resolution of about $5^{\circ}$.

A signal propagation time on the copper readout strips of $6 \pm 1 \mathrm{~ns} \mathrm{~m}^{-1}$ was measured, close to the literature value of $5.6 \mathrm{~ns}$ $\mathrm{m}^{-1}$ taken from [7].

\section{Summary}

The LMU Cosmic Ray Measurement Facility is ready for validation of full-size SM2 quadruplet modules foreseen for the ATLAS New Small Wheel upgrade. All 12280 electronic channels of an SM2 detector can be read out. The analysis of all relevant quantities of a full size pre-series chamber has been presented with emphasis on the reconstruction of the mechanical chamber accuracy using cosmic muons.

\section{References}

[1] ATLAS Collaboration, The ATLAS Experiment at the CERN Large Hadron Collider, JINST 3 S08003 (2008).

[2] ATLAS Collaboration, New Small Wheel Technical Design Report (2013) CERN-LHCC-2013-006.

[3] Y. Giomataris et al., Micromegas: a high-granularity position sensitive gaseous detecor for high particle-flux environments, Nucl. Instrum. Meth. A376 (1996) $29-35$.

[4] L. Jones, APV25-S1 User Guide, http://cds.cern.ch/record/1069892/files/ cer-002725643.pdf (2001).

[5] S. Martoiu et al., Front-end electronics for the Scalable Readout System of RD51, Nuclear Science Symposium and Medical Imaging Conference (NSS/MIC) (2011) $2036-2038$

[6] ANSYS Inc., ANSYS Mechanical APDL Element Reference, http://148.204.81.206/Ansys/150/ANSYS Meachnical APDL Element Reference.pdf.

[7] D. Nührmann, Das komplette Werkbuch Elektronik, Band 3\&4, Franzis'(1994) 2852. 